D

D

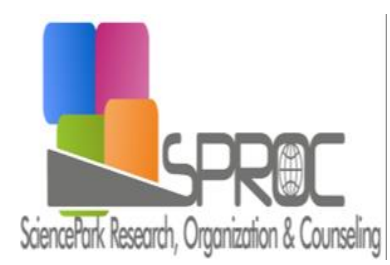

\title{
$R{ }^{d} D$ q Delq $D M$
} $q \quad \mathrm{qD} \quad \mathrm{Clq}$

D $\quad$ 'New Trends

[ and Issues Proceedings

Clq D CID $q$ D on Humanities and Social Sciences

$M \quad D D \quad D \quad D$

$D$

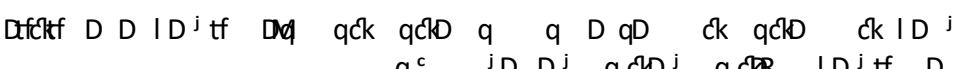
$q^{c} \quad{ }^{j} D \quad D^{j} \quad q C K D^{j} \quad q C R R \quad I D^{j}$ tf $\quad D$

D

$$
\begin{aligned}
& D^{\mathrm{K}} D \quad{ }^{\circ} D \quad \text { I } q \text { CRD } q^{\mathrm{c}} \quad \mathrm{j}^{\mathrm{D}} \mathrm{D} \quad \mathrm{j}^{\mathrm{j}} \\
& D^{\square} D \quad{ }^{\circ} D \quad \text { I } q \quad C D q^{c} \quad{ }^{j} D \quad{ }^{j} D
\end{aligned}
$$

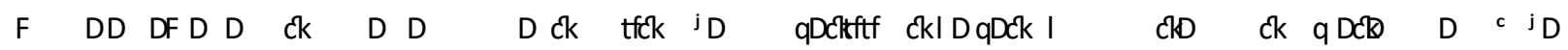
ck D Dck ck q DNew Trends and Issues Proceedings on Humanities and Social SciencesD q $q$ DD

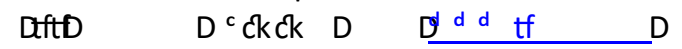

D

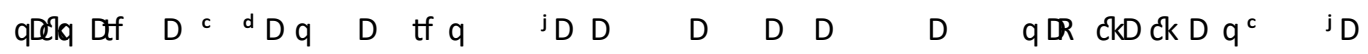

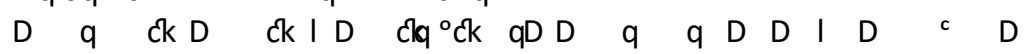

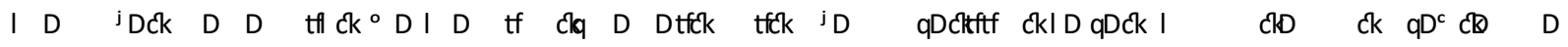

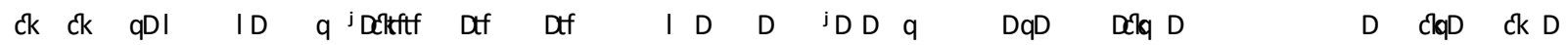

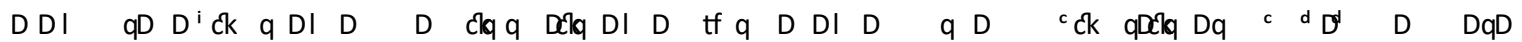

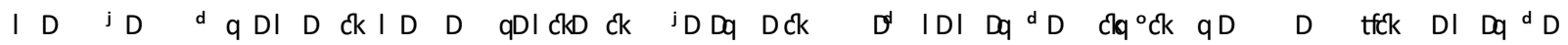

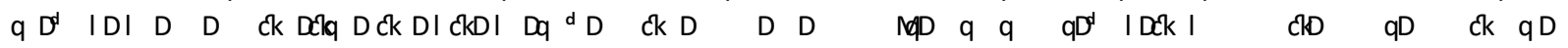

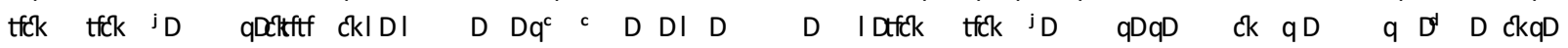

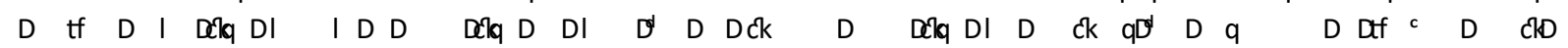
Ckqck j D

jd Dek I ClD Ck quffk tfek jD qD DCk ck qDD 


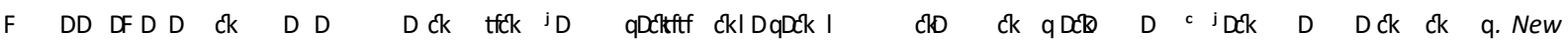

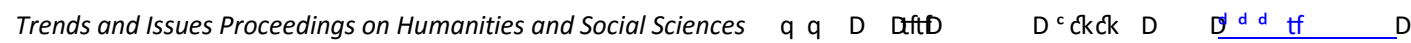

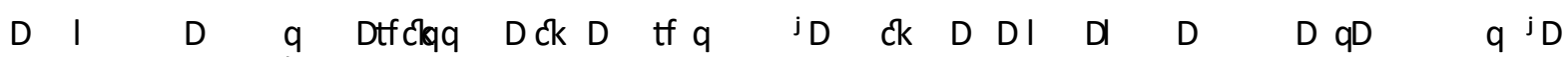

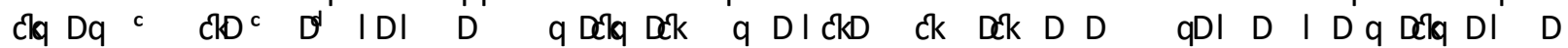

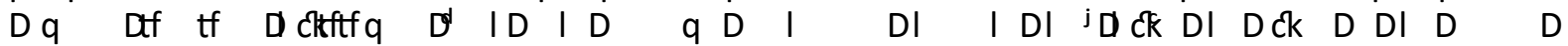
$D \quad D q^{c} \quad q \quad q D q D$ I $\quad D|\quad D \quad c k c k q D \quad D \quad c k \quad D| c k D|\quad| D \quad D$ MDck I $\quad D$

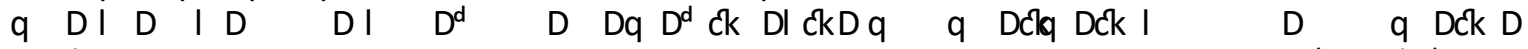

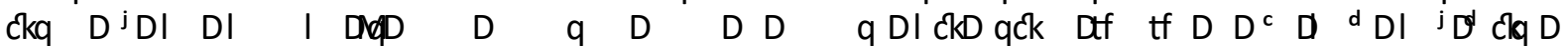
$D^{c} \quad D q \quad C k D D^{j}$ q $D$ D ck $D \quad$ tfckD $c k D$

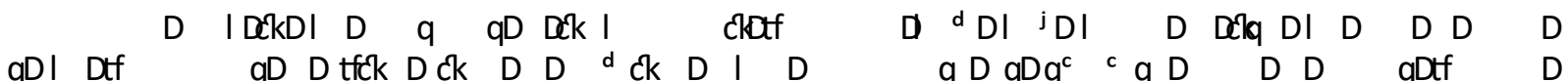

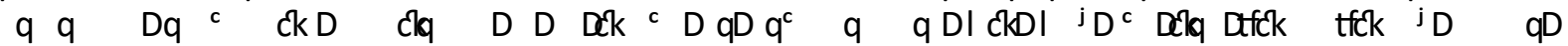

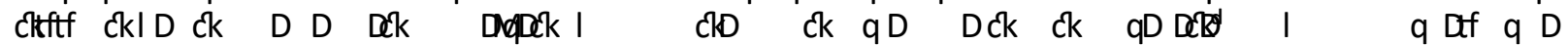

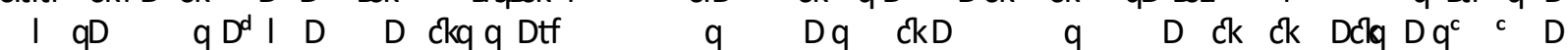

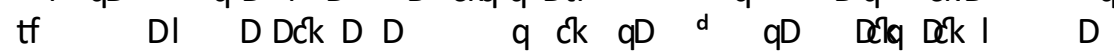

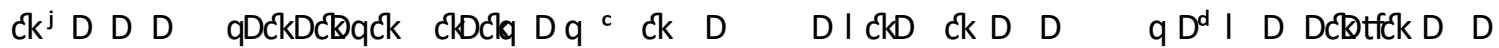

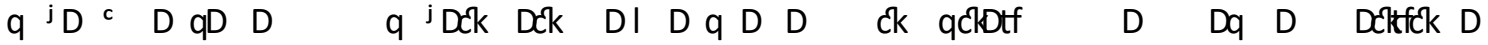

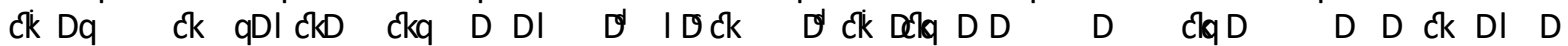

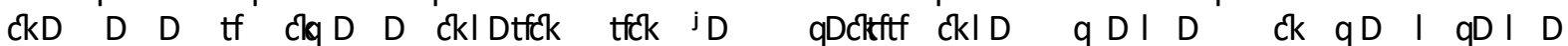

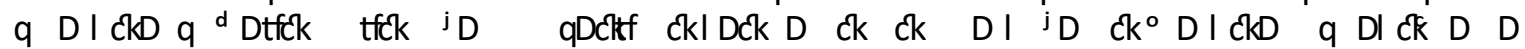

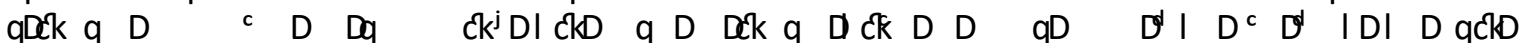

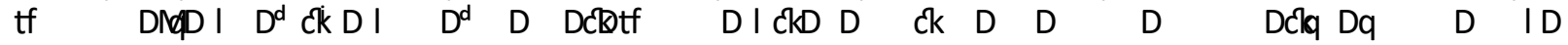

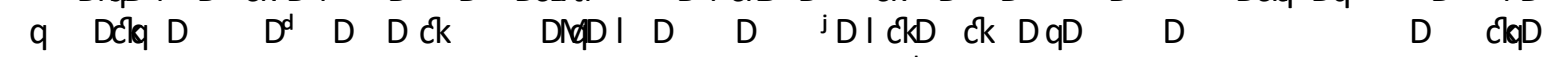

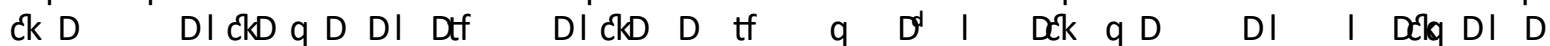

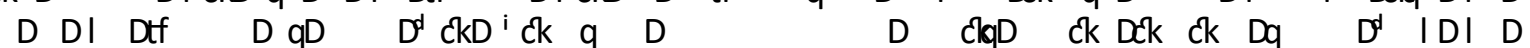

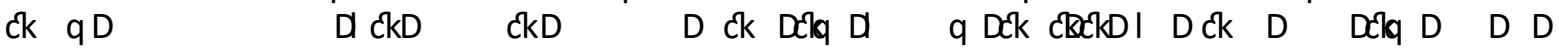
ClqD Clq Ck qDf $\quad D$

D
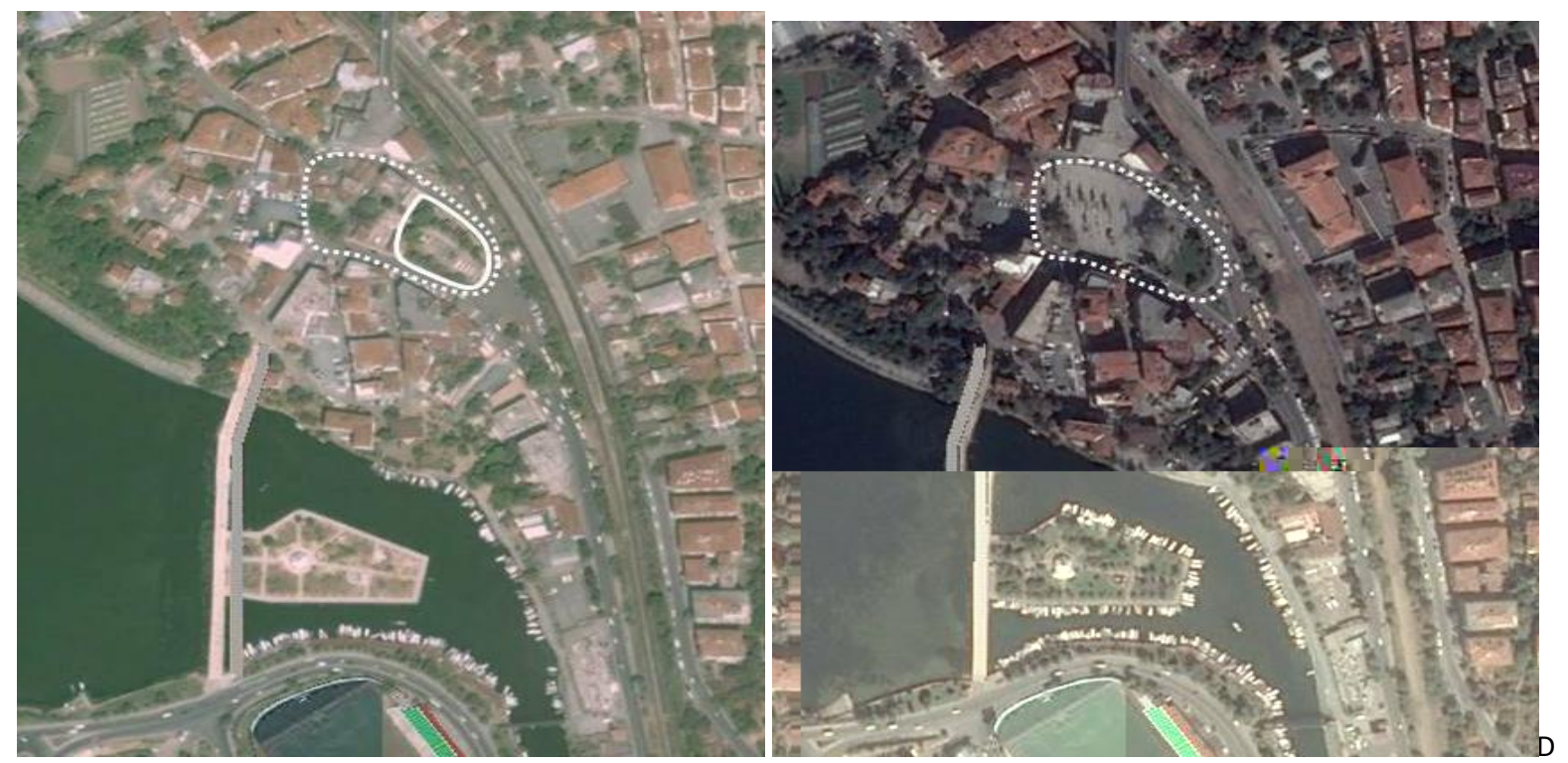

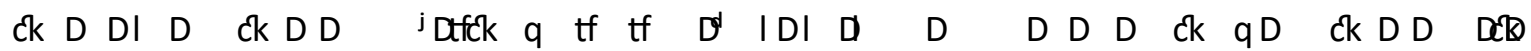

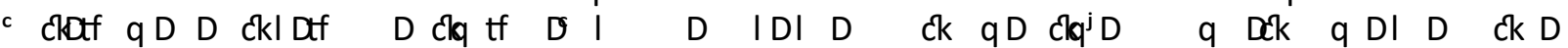

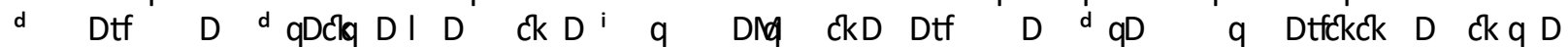

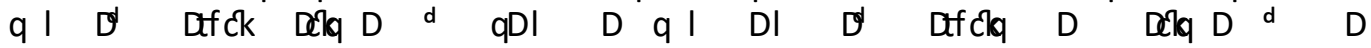

I q $D$ I ckD ckq q D $D$ tf $q$ Dck $D$ D ck $D \quad q D \quad D \quad c k q D$ q I $D$ Dck $c k D$ $q^{j}$ DCk CBClq $D$ D q ${ }^{c} c^{c k} D^{d} \quad D \quad D \quad q$ ckq DM DCk Dtf tf DI ckDtf clq D ck DCD 

$F \quad D D \quad D F D D$
$F$

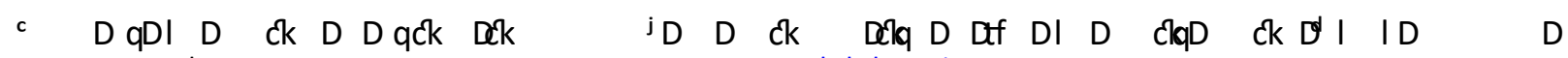

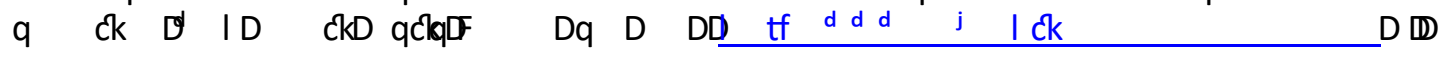

$\mathrm{D}$

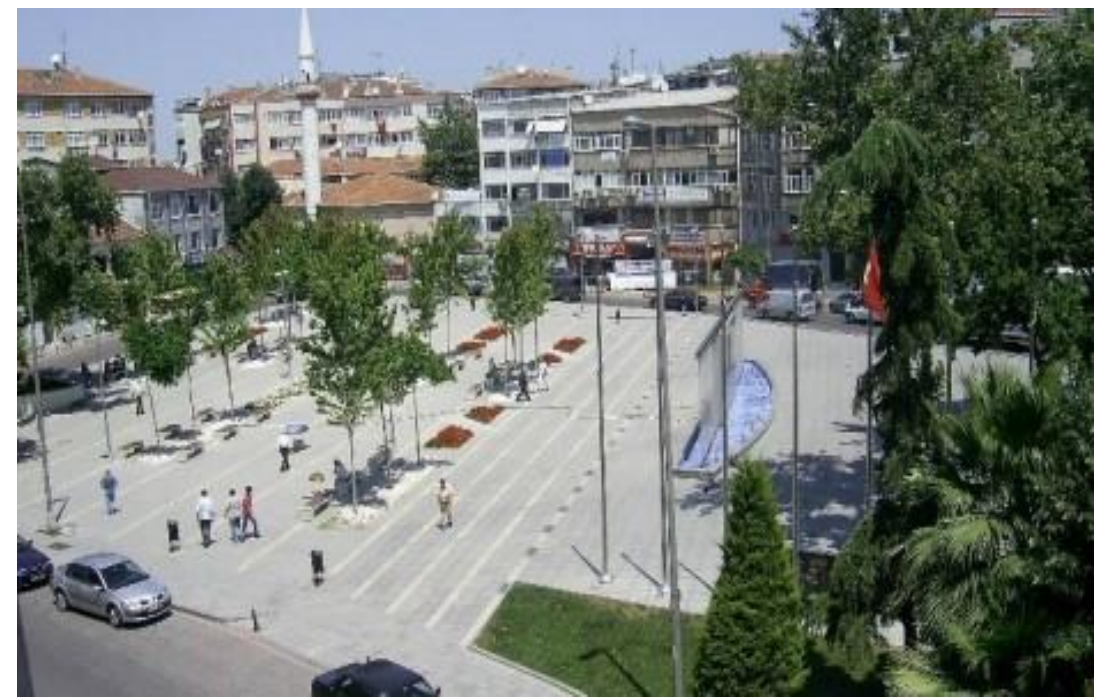

$\begin{array}{llllllllllll} & D \\ 1\end{array}$ tf $q$ Ck $q D$ tf $q \mathbb{D}$

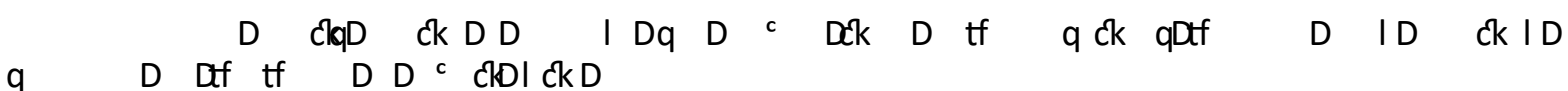
D I I Df $D$ ckq D D $D$

$D \quad q q \quad q D \quad d \quad q D \quad$ Leqq $D$ ck $\quad D \quad$ ck $D$

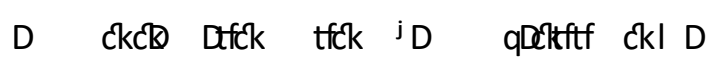

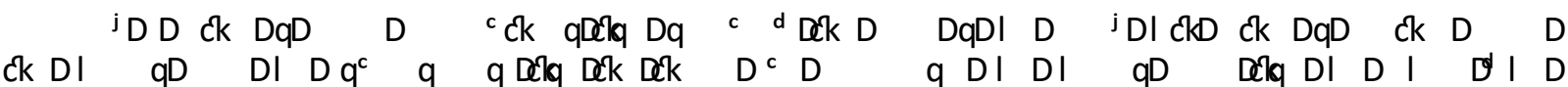

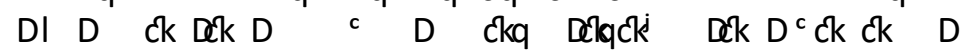

\subsection{Interview}

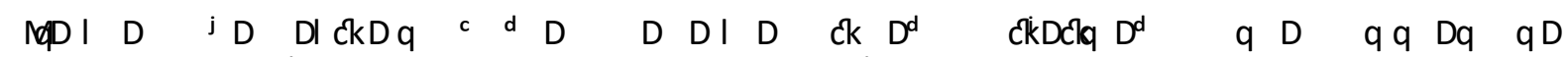
c q q DM DCk G ID af tf $D$ D DI Dek D $\quad$ I I D tf q D DqDI D ck DD DI Dek

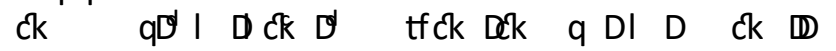

na c d $D \quad q$ Dek $D$

$\begin{array}{lllllllllll}D & { }^{j} D & D & D & D I & D & C k & D\end{array}$

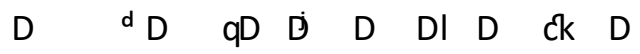

$D \quad D \quad I \quad I D q D D \quad D \quad D$

D D Df $\quad D$ Lffk $D^{j} D I \quad D \quad c k \quad D$

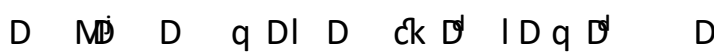

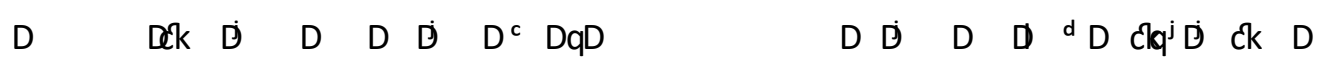

\subsection{Evaluation of Interview}

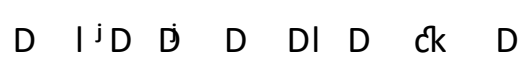




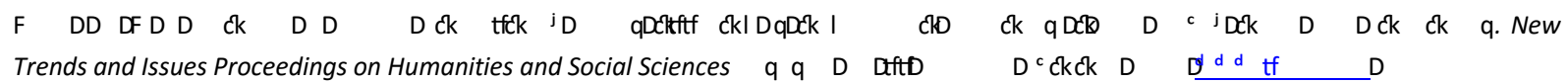

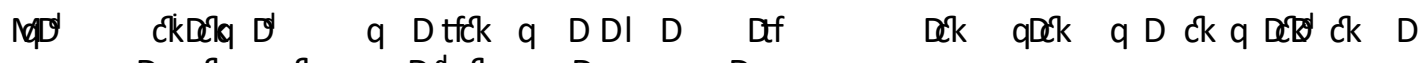
$q \quad D \quad c k \quad c k \quad q \quad D^{d}$ ck $q \quad D \quad q \quad q \quad D$

d $D \quad$ QD $\quad D \quad D \quad$ DI $D$ Ck $\quad D$

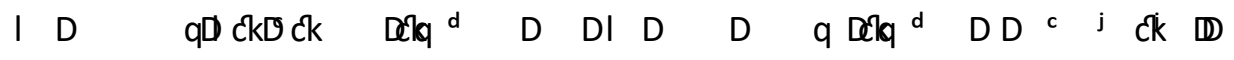
$q^{d} \quad$ Dk $\quad D \quad D$

D

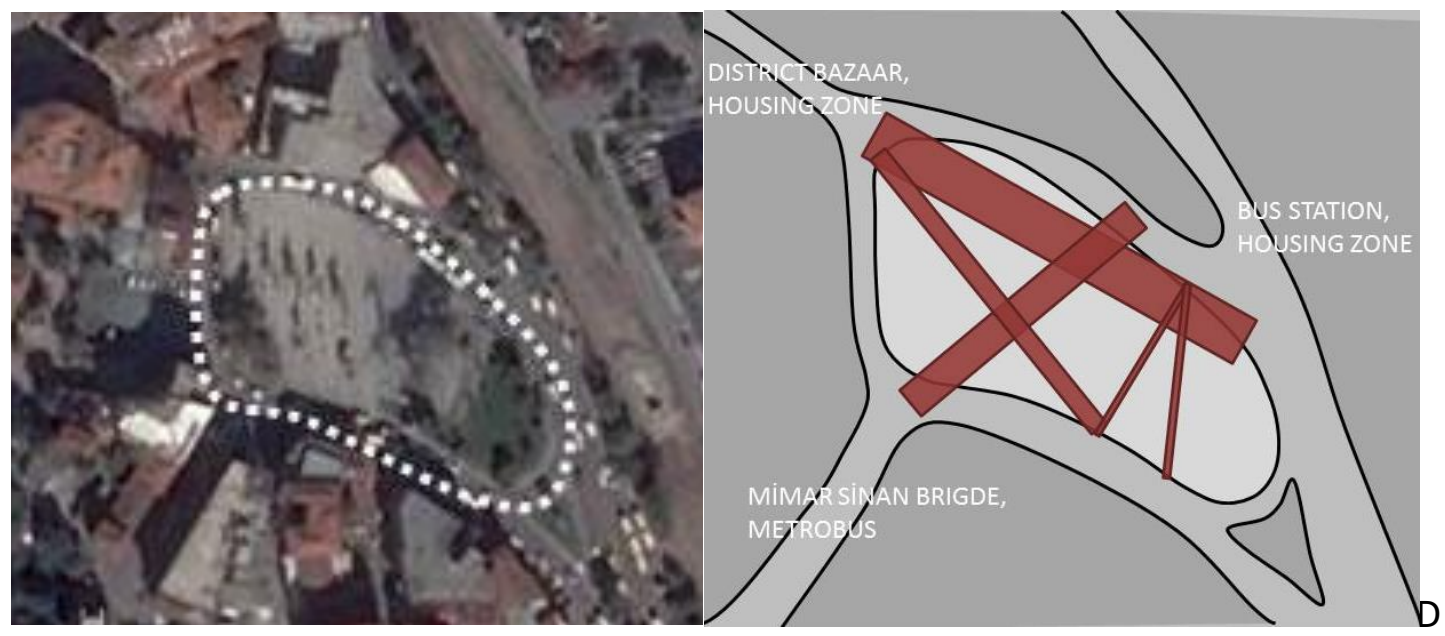

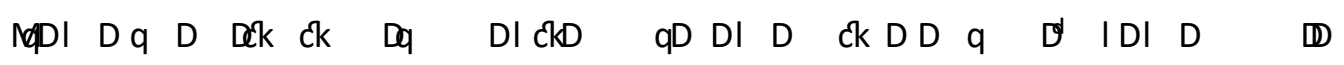
D af $D$ Qffk $D^{\mathrm{j} D I} \mathrm{D} \quad \mathrm{Ck} \quad \mathbb{D}$

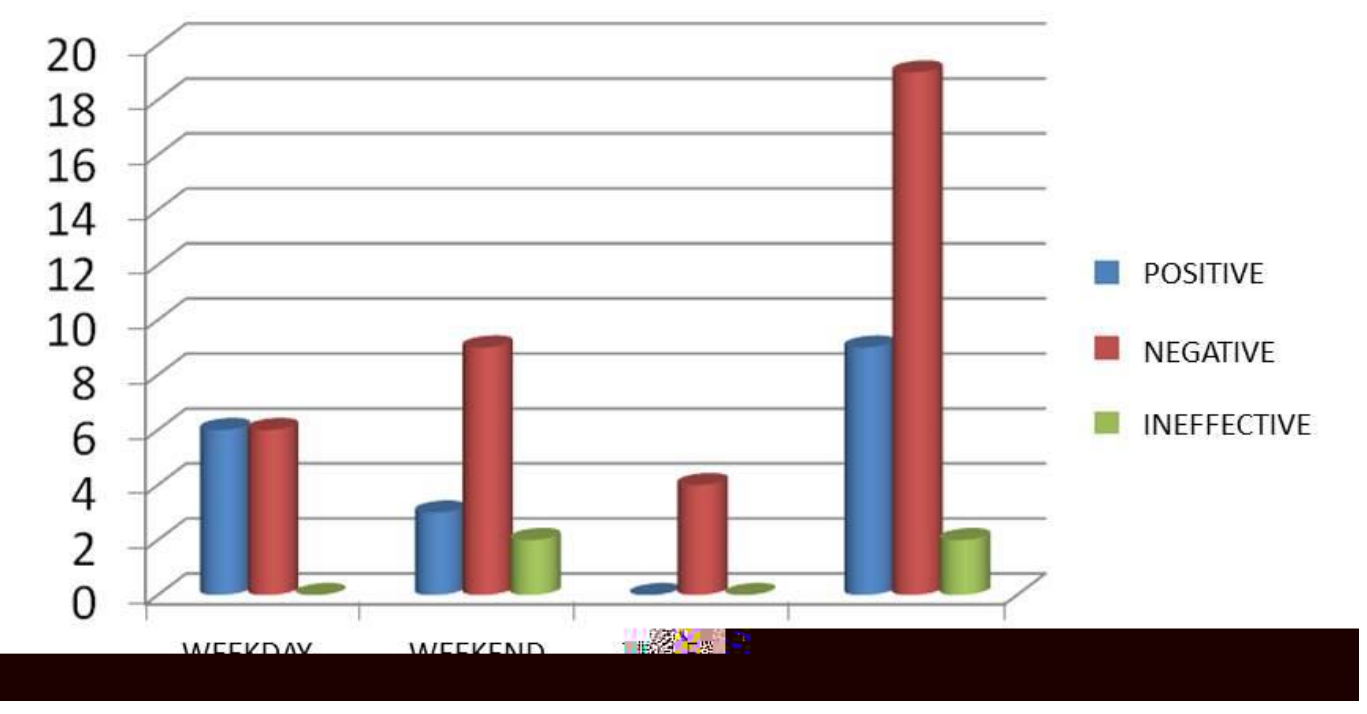

$M D \quad D \quad q \quad D I \quad D \quad c k$ G IDq $\quad$ G $\quad D$

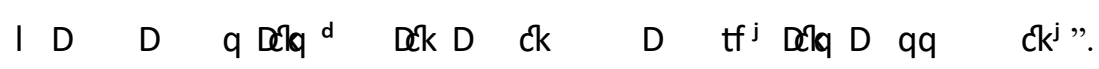




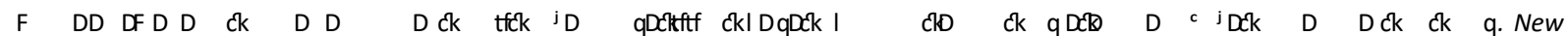

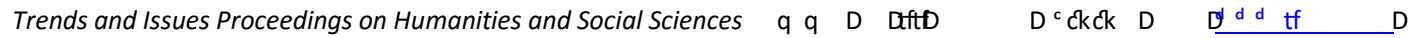

\subsection{Observation}

M D DI CkD

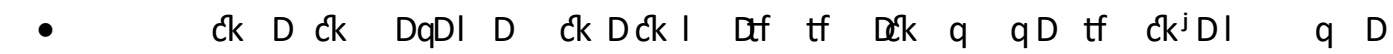



D

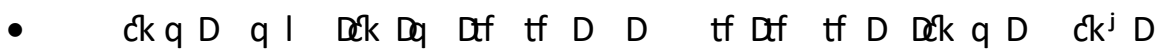

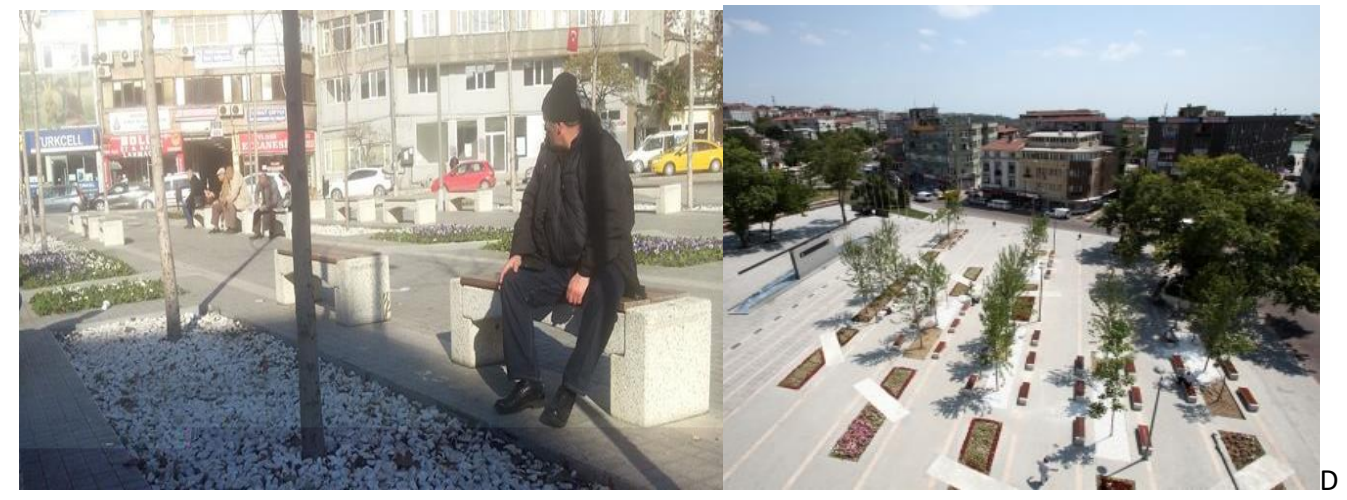

- $\quad c k$ D D ckj $\quad$ ek q DI D ck DekD .

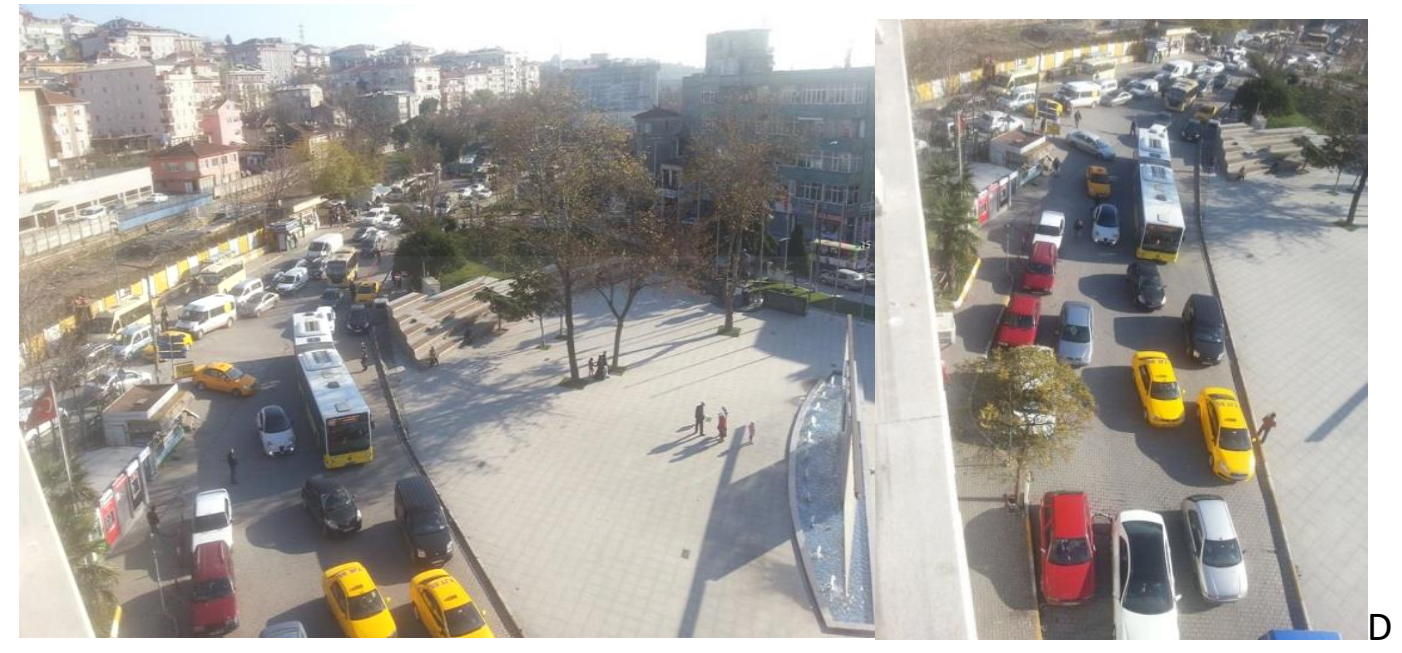




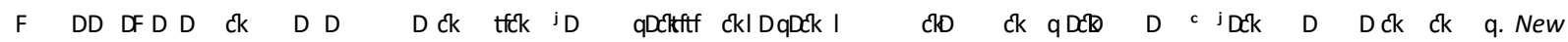

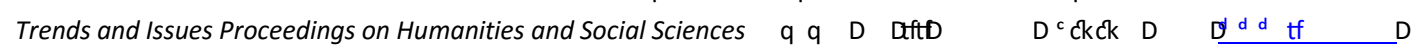

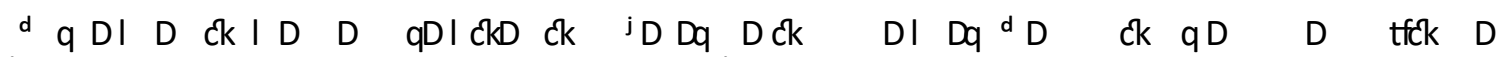
I Dq ${ }^{d} \mathrm{D}$ q Delq DI D $D$ Ck Delq DCk DI CKDI Dq ${ }^{d} \mathrm{D}$ ck D

D D

D

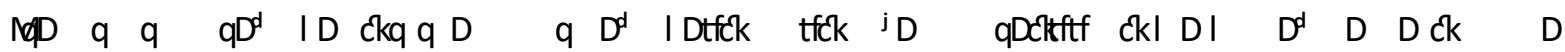

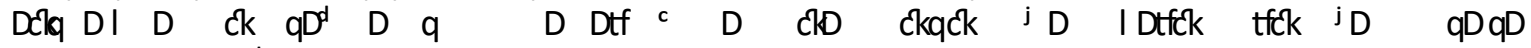

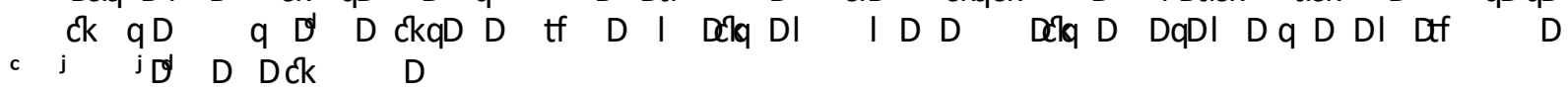

F D D D Dimarlikta Katilimci Tasarim Yaklasimlarinin Analizi Ve Kucukcekmece Orneginde

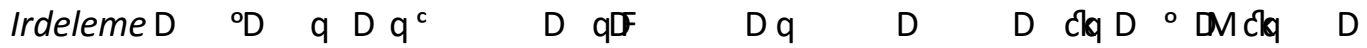

ck ck qDR D D D owards a New Professional Role", Sanoff, H. (Ed.), Participatory Design, Theory and Techniques, North Carolina D

Clq D D DMimarlik Egitiminde Topluma Donuk Olma Ilkeleri, Mimarlik Dergisi D ～D D ck D D DMimarlikta Gorsel Etki Degerlendirme Yontem ve Teknikleri, Yildiz Teknik Universitesi Basim

D 